\title{
JOURNAL.RU
}

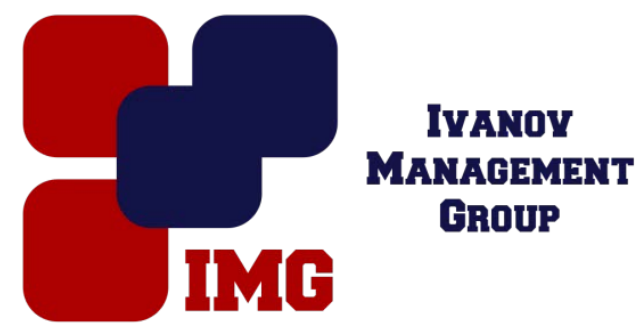

Герасименко Е.Ю., Мерабян М.А., Переходов М.В.

Донской Государственный Технический Университет

Ростов-на-Дону, Россия

doi: 10.18411/lj-30-11-2016-3-03

idsp 000001:lj-30-11-2016-3-03

\section{Определение электро-кинетических параметров электро-химической системы при замедленном разряде}

\section{Аннотация}

В данной работе определяются электро-кинетические параметры электрохимической системы при замедленном разряде, были получены значения идентифицируемых параметров.

Ключевые слова: Двухэлектродная электрохимическая система, плоскопараллельные электроды, математическая модель.

В данной работе исследуется двухэлектродная электро-химическая система

$$
\mathrm{Cd}\left|(0,4 \div 0,75) \mathrm{M} \quad \mathrm{Na}_{2} \mathrm{SO}_{4}+5 \cdot 10^{-3} \mathrm{M} \quad \mathrm{CdSO}_{4}\right| \mathrm{Cd}
$$

с равными плоско-параллельными электродами площадью $\mathrm{S}$, удаленными друг от друга на расстояние l. Изучение системы производится при выключении ее на постоянное напряжение $U_{i}, \mathrm{i}=\overline{1 ;}$.Далее фиксируется установившееся значение тока Іів цепи этой системы. При этом считается, что кинетика электродных процессов контролируется стадией разряда-ионизации. Математической моделью электрохимических процессов в рассматриваемой системе является следующая система уравнений:

$$
\begin{gathered}
I=s i_{0}\left(\exp \left(-\frac{\alpha z F \eta_{k}}{R T}\right)-\exp \left(+\frac{(1-\alpha) z F \eta_{k}}{R T}\right)\right) ;(1) \\
I=s i_{0}\left(\exp \left(-\frac{\alpha z F \eta_{a}}{R T}\right)-\exp \left(-\frac{(1-\alpha) z F \eta_{k}}{R T}\right)\right) ; U=\eta_{a}-\eta_{k}+I R_{\ni}(2),
\end{gathered}
$$

где i0- ток обмена , $\alpha$ - число переноса, $\mathrm{z}$ - валентность реакции, $\eta_{a}$ - поляризация анода, $\eta_{k}$ - поляризация катода, $R_{\ni}$ - сопротивление электролита, $\mathrm{U}$ - питающее напряжение, I - ток во внешней цепи. В модели (1)-(3) идентифицируются параметры $\alpha, z, U_{0}$. 
При заданном токе I из уравнений (1) и (2) могут быть найдены (численно) поляризации $\eta_{k}$ и $\eta_{a}$. Обозначим:

$$
\eta_{k}=f_{1}\left(I ; \alpha ; z ; i_{0}\right), \eta_{a}=f_{2}\left(I ; \alpha ; z ; i_{0}\right) .(4),(5)
$$

Подставив (4) и (5) в (3), получим

$$
U=f_{2}\left(I ; \alpha ; z ; i_{0}\right)-f_{1}\left(I ; \alpha ; z ; i_{0}\right)+I R_{\ni} .
$$

Применим к последней формуле метод наименьших квадратов. После этого можно записать выражение для суммарной квадратичной ошибки эксперимента

$$
\Phi\left(\alpha ; z ; i_{0}\right)=\sum_{i=1}^{M}\left(f_{2}\left(I_{i} ; \alpha ; z ; i_{0}\right)-f_{1}\left(I_{i} ; \alpha ; z ; i_{0}\right)+I_{i} R_{\ni}-u_{i}\right)^{2} .
$$

Введем обозначение $W_{i}\left(\alpha ; z ; i_{0}\right)=f_{2}\left(I_{i} ; \alpha ; z ; i_{0}\right)-f_{1}\left(I_{i} ; \alpha ; z ; i_{0}\right)+I_{i} R_{\ni}$, после чего получаем

$$
\Phi\left(\alpha ; z ; i_{0}\right)=\sum_{i=1}^{M}\left(W_{i}\left(\alpha ; z ; i_{0}\right)-u_{i}\right)^{2} .
$$

Минимизацию функции $\Phi\left(\alpha ; z ; i_{0}\right)$ будем вести методом Ньютона с помощью итерационной формулы [1]

$$
\begin{gathered}
\Phi\left(\alpha^{(k+1)} ; z^{(k+1)} ; i_{0}^{(k+1)}\right)= \\
=\sum_{i=1}^{M}\left(W_{i}\left(\alpha^{(k)} ; z^{(k)} ; i_{0}^{(k)}\right)+\frac{\partial W_{i}}{\partial \alpha}\left(\alpha^{(k)} ; z^{(k)} ; i_{0}^{(k)}\right) \Delta \alpha^{(k)}+\right. \\
\left.+\frac{\partial W_{i}}{\partial z}\left(\alpha^{(k)} ; z^{(k)} ; i_{0}^{(k)}\right) \Delta z^{(k)}+\frac{\partial W_{i}}{\partial i_{0}}\left(\alpha^{(k)} ; z^{(k)} ; i_{0}^{(k)}\right) \Delta i_{0}^{(k)}-u_{i}\right)^{2}
\end{gathered}
$$

где $\mathrm{k}$ - номер итерации; $\alpha^{(k)}, z^{(k)}, i_{0}^{(k)}$ - шаги на $\mathrm{k}$-ойитерации. Выбор шагов $\alpha^{(k)}, z^{(k)}$ и $i_{0}^{(k)}$ для $\mathrm{k}$-ойитерации производится из условий

$$
\begin{aligned}
& \frac{\partial \Phi\left(\alpha^{(k+1)} ; z^{(k+1)} ; i_{0}^{(k+1)}\right)}{\partial \Delta \alpha^{k}}=0 ; \frac{\partial \Phi\left(\alpha^{(k+1)} ; z^{(k+1)} ; i_{0}^{(k+1)}\right)}{\partial \Delta z^{k}} \\
= & 0 ; \frac{\partial \Phi\left(\alpha^{(k+1)} ; z^{(k+1)} ; i_{0}^{(k+1)}\right)}{\partial \Delta i_{0}{ }^{k}}=0
\end{aligned}
$$

Если ввести символическое обозначение $\bar{X}^{k}=\left(\alpha^{(k)} ; z^{(k)} ; i_{0}^{(k)}\right), \quad$ то последняя система в развернутом виде окажется такой: 


$$
\left\{\begin{array}{l}
\sum_{i=1}^{M}\left(W_{i}\left(\bar{X}^{k}\right)+\frac{\partial W_{i}}{\partial \alpha}\left(\bar{X}^{k}\right) \Delta \alpha^{(k)}+\frac{\partial W_{i}}{\partial z}\left(\bar{X}^{k}\right) \Delta z^{(k)}+\frac{\partial W_{i}}{\partial i_{0}}\left(\bar{X}^{k}\right) \Delta i_{0}{ }^{(k)}-u_{i}\right) \frac{\partial W_{i}}{\partial \alpha}\left(\bar{X}^{k}\right)=0 \\
\sum_{i=1}^{M}\left(W_{i}\left(\bar{X}^{k}\right)+\frac{\partial W_{i}}{\partial \alpha}\left(\bar{X}^{k}\right) \Delta \alpha^{(k)}+\frac{\partial W_{i}}{\partial z}\left(\bar{X}^{k}\right) \Delta z^{(k)}+\frac{\partial W_{i}}{\partial i_{0}}\left(\bar{X}^{k}\right) \Delta i_{0}{ }^{(k)}-u_{i}\right) \frac{\partial W_{i}}{\partial z}\left(\bar{X}^{k}\right)=0 \\
\sum_{i=1}^{M}\left(W_{i}\left(\bar{X}^{k}\right)+\frac{\partial W_{i}}{\partial \alpha}\left(\bar{X}^{k}\right) \Delta \alpha^{(k)}+\frac{\partial W_{i}}{\partial z}\left(\bar{X}^{k}\right) \Delta z^{(k)}+\frac{\partial W_{i}}{\partial i_{0}}\left(\bar{X}^{k}\right) \Delta i_{0}{ }^{(k)}-u_{i}\right) \frac{\partial W_{i}}{\partial i_{0}}\left(\bar{X}^{k}\right)=0
\end{array}\right.
$$

Выполняя решение данной системы, находят шаги $\alpha^{(k)}, z^{(k)}, i_{0}^{(k)}$, а далее с помощью формулы (7) вычисляют $\Phi\left(\bar{X}^{(k+1)}\right)$. Вычислительный процесс продолжается до тех пор, пока не выполнится условие $\left.\mid \Phi \overline{(X}^{(k+1)}\right)-$ $\Phi\left(\bar{X}^{(k)}\right) \mid \leq \varepsilon$, где $\varepsilon$ - допускаемая погрешность.

Для указанной электрохимической системы при $\alpha^{(0)}=0,2 ; z^{(0)}=1 ; i_{0}^{(0)}=$ 7,5 A/м2 были получены следующие значения идентифицируемых параметров: $\alpha=0,4 ; z=2 ; i_{0}=10,5 \mathrm{~A} / \mathrm{M} 2$. Расхождение с известными данными не превосходит $5 \%$.

\section{Литература}

1. Алифанов О.М., Артюхин Е.А., Румянцев С.В. Экстремальные методы решения некорректных задач. М.:Наука, 1998. 